


\title{
5 los registros civiles y la débil institucionalidad
}

\author{
Por Pablo Carías
}

La calidad de los registros civiles, es esencial para formular políticas de población y de ésta con otros aspectos de la vida social y económica de la nación, la calidad de los registros genera confianza en las instituciones nacionales y en los organismos de cooperación internacional; ninguna política se puede trazar con datos incompletos o de dudosa procedencia, datos que refieren por ejemplo, que el subregistro en materia de defunciones Ilegan hasta un 36\% en nuestro país; imposibilitan que los mismos puedan convertirse en una herramienta útil en la elaboración de estrategias para otros propósitos, pero también se convierte en la razón principal por la cual no es difícil establecer los débiles procedimientos en materia de política y recursos asignados a la tarea de los mismos, un hecho como este nos demuestra que en un al to porcentaje las muertes no se registran y si a esto le agregamos las circunstancias en las cuales no siempre se establece la causa exacta de las muertes, al final tenemos que los organismos encargados de la salud, no disponen de la información adecuada para determinar el perfil epidemiológico de la nación. ${ }^{1}$

Con todo y que hay al gunas aproximaciones sobre las deficiencias cuantitativas y cualitativas sobre el registro, es bueno señalar que es difícil establecer con precisión los defectos y sobre todo las estadísticas exactas que nos informen en qué número se deja de registrar los diferentes actos. Los organismos encargado de proporcionar información, especialmente el Registro $\mathrm{N}$ acional de las Personas, no disponen, por diferentes razones, de información sistematizada que sea de utilidad pública.

En el ámbito de lasnaciones latinoamericanas, la Lic. Elizabeth Spehar, C oordinadora Ejecutiva de la U nidad para la Promoción de Ia Democracia de la O rganización de Estados A mericanos O EA, resal taba que como parte del apoyo que la OEA le brinda a ese proceso, "... la U nidad ha identificado la modernización de los registros civiles como objetivo primordial. El registro civil es una institución cuya importancia es reconocida universalmente. Su trascendencia deriva no sólo de la captación de datos para la información sociodemográfica, sino también del hecho de ser la base y piedra angular para la inscripción del ciudadano en el padrón electoral, instancia que inicia y renueva el ejercicio fundamental del derecho al sufragio. Por esto el R egistro Civil esuna institución básica para el ejercicio y la protección de los derechos civiles y políticos". 2

En el debate que se dio en la C onferencia sobre Fortal ecimien- to de los R egistros Civiles celebrada en Panamá a finales de los $90 \mathrm{~s}$ también se dijo por parte del Dr. Edmundo Jarquín, Jefe de la División de Estado y Sociedad Civil del BID , "... el tema de los Registros C iviles adquiere una dimensión que trasciende la tradicional. N o se trata sólo de un tema socio-demográfico, como lo caracterizaba Elizabeth Spehar, ni tampoco únicamente de la consolidación de los sistemas electorales, cuestión fundamental en sí misma; si no que el tema de los registros civiles se articula con problemáticas de fiscalidad, de identificación ciudadana, de ampliación de la base tributaria, de seguridad jurídica; con un conjunto de condiciones elementales en términos de cohesión, de integración, y de inclusión en una sociedad moderna". 3

En H onduras, según un documento del Fondo de Población de Ias $\mathrm{N}$ aciones U nidas (FN U A P), el sistema tradicional de información sobre hechos vitales es deficiente, de la misma forma como sucede en muchos otros países en vías de desarrollo. Existe una gran omisión en el registro de nacimientos, a lo que se debe agregar la inscripción tardía y defectuosa de los mismos, mientras la omisión del registro de muertes es todavía más grave. Se encontró, en una información preliminar, que en Honduras en el período entre 1984 y 1989 el total de muertes registradas fue menos del 40\%, aunque a principios de esa misma década, más del $40 \%$ de las muertes no se registraba y sólo el 13\% de las muertes era certificada por un médico. 0 tro tanto ocurre con los nacimientos, a principios de la década de los cincuenta la omisión era de un $17.7 \%$ mientras que para el período de 1950-1974 en un 15\%. Para principios de la década de los setenta las estimaciones varían de $10.6 \%$ a $27.6 \%$, lo cual es una omisión bastante significativa. ${ }^{4}$

La información anterior es reflejo de los débiles procedimientos que se tienen en el país para la captura de hechos vitales, sin embargo, es más grave cuando se constata que no es fácil construir información fidedigna acerca de los registros, habida cuenta que las fuentes de datos no están actualizados o las oficinas local es y regionales no hacen los reportes correspondientes.

Las estadísticas vitales son esenciales para el análisis de la dinámica de la población de H onduras y su proyección hacia el futuro, en este sentido, los registros civiles son un componente importante en otros tipos de fuentes demográficas; los mismos pueden relacionarse con los $C$ ensos para hacer comparaciones y sacar deducciones que permitan estudios más detallados. Entre un C enso y otro, los 
registros civiles pueden marcar tendencias demográficas importantes, tanto en el ámbito local como nacional.

Según los organismos especializados en el abordaje de esta problemática adscrito a la O rganización Panamericana de la Salud (OPS) y la O rganización M undial de la Salud (OM S), Ios sistemas de registro civil y las estadísticas vitales derivadas de ello proporcionan una base de información amplia e integral en lo referente a la población de un país. C ada sociedad necesita definir las características y las ten dencias en su población. U no de los usos más importantes de las estadísticas vitales es en los análisis demográficos, un prerrequisito para la planificación y el análisis del desarrollo económico y social.

Las estadísticas vital es se derivan de los datos de registro civil globales; estos últimos deben, por consiguiente, constituir la fuente primaria para los datos confiables, continuos, permanentes y de calidad para unas estadísticas vitales de un país. La documentación de los sucesos vitales tiene gran val or y utilidad en el desarrollo económico y social de los países. También tiene valor inapreciable como prueba de identificación personal y para la protección de los derechosindividuales.

$\mathrm{H}$ oy en día como resultado de los cambios provocados por el modelo de acumulación de capital y de los movimientos de la población, han surgido una serie de fenómenos que requieren la atención de los organismos del registro de las personas; me refiero a aspectos relacionados con el tráfico de personas, identificación de migrantes y hasta personas vinculadas con el crimen organizado.

$\mathrm{H}$ asta ahora, uno de los problemas graves de los registros civiles en $\mathrm{H}$ onduras es su base legal, según las normas establecidas, los registros los realiza el R egistro $\mathrm{N}$ acional de las Personas; entidad que depende del Tribunal $\mathrm{N}$ acional de Elecciones, mismo que está integrado con representantes de los partidos políticos legalmente reconocidos, de esta manera, el registro queda en manos de los políticos, por lo cual, una tarea que por su propia natural eza debería de estar al margen de las contingencias político- partidistas, queda supeditada a las decisiones de estas instituciones cuya función no tiene nada que les relacione con tareas de este tipo. Los registros civiles de una nación; si bien es cierto deben de obedecer a la política estatal, los mismos deben estar al margen de las políticas sectarias que tradicionalmente ha inspirado a las organizaciones partidistas en nuestro país, por lo menos así lo atestigua su práctica en más de un centenar de años.

$\mathrm{O}$ tro elemento que hay que destacar es que en $\mathrm{H}$ onduras, como parte de las diversas carencias que sufre la nación, su población no ha logrado apoderarse de una cultura que favorezca la realización de los registros civiles en tiempo oportuno. H ay necesidad de impulsar en el sistema educativo nacional, un aspecto que incluya una formación en ese sentido, donde se destaque la necesidad ciudadana de efectuar todos los registros que tiene el país, de igual manera la legislación hondureña necesita una actualización histórica, a fin de que se proporcionen los estímulos y sanciones co- rrespondientes en materia de registros.

La transición demográfica, proceso a la cual el país ha entrado en las últimas dos décadas, requiere de registros que nos permitan hacer las evaluaciones periódicas para determinar la asignación de recursos en áreas tan sensibles como la educación, vivienda, salud y seguridad social. La transición demográfica es algo dinámico que tiene incidencia en otros componentes de la sociedad en su conjunto, de ahí la necesidad de estudiar los hechos vitales.

En el país no se han hecho estudios sobre los registros civiles con una perspectiva precisa acerca de las debilidades que han mostrado y las posibilidades de mejorarlos, los estudiosque se han hecho han tenido otra finalidad; en algunos casos se han realizado con la idea de conocer al gunos hechos de los registros vitales desde el punto de vista histórico, han sido estudios sobre casos parciales y no como al go sistemático. Esta es una tarea pendiente.

Los logros obtenidos en otros países, cuya experiencia, en gran parte son recogidos, por los organismos especial izados de las $\mathrm{N}$ aciones $U$ nidas $O N U$, en diferentes libros, boletines y revistas del organismo mundial, nos permiten, por un lado hacer un balance comparativo, pero también nos posi bilita visualizar las experiencias de otras naciones como posibles para $\mathrm{H}$ onduras.

Una propuesta de mejora de los registros civiles

Si los resultados finales de la institución responsable de los registros civiles son deficientesy existela percepción de al gunosfuncionarios y exfuncionarios que el personal encargado de llevar las tareas regístral es muestran un bajo nivel de calificación e idoneidad, entrar en una fase de análisis de su personal con vista a determinar el impacto que esto tiene en la eficiencia de los registros, carece de sentido y los resultados que pudieran derivarse de una análisis semejante, son claramente visibles.

Si bien es cierto, en las actuales condiciones en las que por el propio debilitamiento de las funciones del Estado, Ios asuntos relacionados con la demografía no son de una gran prioridad, la existencia de las viejas necesidades de contar con datos actual izados sobre los registrosy el aparecimiento de nuevos problemasque con el tiempo se van agudizando como ser el tránsito de indocumentados, tráfico de niños y hechos delictivos, deberían hacer crecer el interés de organismos internacionales y de los gobiernos por el tema propuesto.

El "pegado original" de las múltiples deficiencias en la captura, sistematización y aprovechamiento de los registros civiles para un mejor desempeño de la función pública está en la politización partidaria de la cual han sido victimas, no obstante, esto no quiere decir que por el simple hecho de cambiar la actual estructura en la dirección de los registros, inmediatamente las cosas van a cambiar, no será así, el nuevo sistema tendrá un período de reordenamiento, mismo que requerirá también de un continuado esfuerzo por aumen- 
tar los recursos que una tarea de estas requiere.

De una situación en la cual los registros están en manos de los partidos políticos, a una nueva en la cual se despolitizan los mismos, deberá de darse un período de transición, mismo en el cual se deberá hacer una evaluación de todo su personal por una firma independiente para garantizarle al personal que se considere capacitado en el desempeño de sus funciones su estabilidad en sus puestos.

La nueva legislación en materia de registros civiles debe ser precisa en la definición de conceptos, en ese mismo sentido debe de haber una armonización en todas las leyes que tengan referencia a éstos para evitar en lo posible contradicciones que vuelven confusa la interpretación de las normas.

En la propuesta de mejoramiento, es importante tomar en cuenta las experiencias de otros países recogidas por las $\mathrm{N}$ aciones $\mathrm{U}$ nidas, según este organismo mundial, el establecimiento de un Comité $\mathrm{N}$ acional de Coordinación Interinstitucional constituido por departamentos que se ocupan de cuestiones de población, salud, registro civil, estadísticas, justicia y otros, es un medio útil de abordar las cuestiones relacionadas con el mejoramiento y la producción de estadísticas vitales. Según la O N U, para que el comité sea eficaz, debe de tener carácter permanente, tener personería jurídica y su personal gozar de estabilidad laboral, excepto por asuntos que las leyes de trabajo prescriben.

En el caso de Honduras, atendiendo a su propia experiencia se trataría de aplicar un sistema centralizado, donde las oficinas municipales dependan directamente de una oficina central. En este sistema las oficinas regional es pierden sentido, no deben de aparecer en una futura legislación, esto tiene aún mayor validez si el registro está tecnificado y existen facilidades para la remisión de la información. Se trata de eliminar todas aquellas instancias burocráticas que duplican funciones y que aumentan los costos de operación.

La nueva estructura de la entidad respon sable de los registros, deberá estar orientada a la creación de un Sistema de R egistro Civil con una oficina central, con relación directa al Sistema $\mathrm{N}$ acional de Estadística y su oficina nacional, la relación se llevará a la práctica por medio del Comité $\mathrm{N}$ acional de Coordinación.

El Director, según nuestra propuesta, esta función debe de cumplirla un Comisionado de los Registros Civiles y debe reunir los siguientes requisitos: ser hondureño por nacimiento, del estado seglar, poseer titulo universitario en el ámbito de postgrado afín a la actividad que va realizar, tener experiencia profesional o académica, no tener juicios pendientes con la justicia ni haber sido juzgado en los tribunales correspondientes y de reconocida honorabilidad. En ningún caso la persona que cumpla esta función tiene que estar cumpliendo funciones en la dirección de al gún partido político ni tener una militancia reconocida en el activismo partidario. Su nombramiento debe hacerse en el $\mathrm{C}$ ongreso $\mathrm{N}$ acional de la República en votación de las dos terceras partes en consulta con la sociedad civil, el Director debe de gozar de inamovilidad en el cargo, salvo limitaciones que señalen las leyes de la nación.
Debe haber una definición de lo que son en sí los registros, que según las $\mathrm{N}$ aciones $\mathrm{U}$ nidas se definen como la inscripción obligatoria, continua y permanente de los hechos vitales ocurridos y de sus características con arreglos a las determinaciones legales de cada país. Es importante según las recomendaciones del organismo mundial, al cual hemos hecho referencia que se establezca con claridad el carácter obligatorio como algo esencial para el buen funcionamiento, de no hacerse, sin sanciones concretas se dice, el aspecto legal pierde todo sentido.

El fenómeno migratorio y los problemas que se derivan de ello, al gunos problemas delictivos como el robo de niños, narcotráfico y otros delitos van permitiendo visual izar la necesidad de ir creando algún tipo de información que sea parte de convenios entre países para uniformar la misma. Se debe de eval uar la posibilidad de registrar alguna característica física de fácil verificación para establecer la identidad de las personas desde su nacimiento.

$\mathrm{H}$ ay opiniones calificadas que recomiendan que la inscripción de los sucesos vitales tal y como lo prescribe la ley en varios países, esté libre de cargas para el declarante, si cumple las disposiciones legales con respecto al tiempo. Recomendación que no excluye la imposición de una tasa por una inscripción tardía, a las inscripciones efectuadas después de haber expirado el plazo establecido, 0 a las búsquedas en los registros y a la certificación de copias auténticas.

Un aspecto a tomar en cuenta debe ser la necesidad de establecer como norma que aquel ciudadano que solicite su Tarjeta de I dentidad debe de presentar la C ertificación de A cta de $\mathrm{N}$ acimiento sólo cuando de al guna manera haya sido verificada en la autoridad central competente, para al gunos entendidos el otorgamiento de la Tarjeta con un documento cuya autenticidad sólo la establece un facsímil no es suficiente, es más, las leyes del país no establecen que una firma en estas condiciones sea suficiente para otorgar legitimidad.

Es importante también que la Ley determine cual ha de ser el criterio que se va a utilizar para hacer el registro de un suceso vital, 0 se establece el lugar de acaecimiento del hecho o el lugar de residencia o se utilizan ambos criterios, en nuestra legislación eso no está claro.

U na de las mayores dificultades que tiene nuestro sistema de registro, es que no tien e la forma adecuada, dada las precarias condiciones en que se desenvuelve nuestro sistema de sal ud al no cubrir el sistema todo el universo de la población, es que esta última circunstancia no queda registrada, sobre todo en las áreas rurales del país, donde la muerte fetal queda como "nació muerto", sin poder con esta información sacar información para determinar las causas de muerte.

Un aspecto importante que señalan las recomendaciones de las $\mathrm{N}$ aciones $\mathrm{U}$ nidas es $\mathrm{l}$ o referente a los permisos de inhumación 0 enterramiento, los mismos dicen las recomendaciones no podrán tener lugar antes de las 24 horas después de haber ocurrido el hecho, ni después de 48 horas desde el mismo. En este sentido, también es 
importante la recomendación que el traslado de los cadáveres debe de hacerse solamente con la autorización de una autoridad sanitaria, aunque el lugar del mismo sea de acuerdo al permiso que otorgue el Registrador, aunque se hace la previsión en el sentido que cuando las muertes se produzcan en circunstancias excepcional es como epidemias, guerras, peligro de contagio u otras, se podrán tomar medidas especial es, siempre con la autorización de las autoridades de sanidad. Estos son aspectos que deberán ser recogidos por una futura reforma a la legislación en el país.
U na de las dificultades que se presenta en la administración del acto del matrimonio, es que, la autoridad que celebra el matrimonio no lo registra, como parte de la centralización de los registros civiles, el matrimonio para que surta los efectos legales, deberá registrarse en el Registro Civil, sin embargo quien los lleva a cabo es el A Ical de o en su defecto un $\mathrm{N}$ otario Público, aquí de lo que se trata es de facilitar los reportes de las alcal días a los R egistros C iviles. 